\title{
Inhibition of Bacterial Adherence on the Surface of Stents and Bacterial Growth in Bile by Bismuth Dimercaprol
}

\author{
HONGJUN ZHANG, PhD, MD,* JAVON TANG, BS, $\dagger$ XANGWEN MENG, PhD, MD,* \\ JACKIE TSANG, $\ddagger$ and TAT-KIN TSANG, MD*
}

\begin{abstract}
Bacterial infection and biofilm formation on the surface of biliary stents is believed to be one of the main factors in stent occlusion. This study explored the role of the new reagent, bismuth dimercaprol, in preventing bacterial adherence and bacterial biofilm formation on the surface of biliary stents. Sterile porcine bile preparations, infected separately with Escherichia coli, Klebsiella pneumoniae, Enterobacter, and Enterococcus, were used as the perfusion media in an in vitro perfusion system. The bacterial growth in the media and the bacterial adherence on the surface of stents were tested when different concentrations of bismuth dimercaprol were used in the perfusion media. BisBAL $(5 \mu M)$ did not inhibit the growth of any of the tested bacterial species. It did, however, significantly decrease the amount of bacteria adhering to the surface of stents for all bacterial strains except Escherichia coli. Bismuth dimercaprol $(20 \mu M)$ significantly inhibited the growth of Escherichia coli, Klebsiella pneumoniae, and Enterobacter and, thereby, significantly decreased the amount of these bacteria adhering to the surface of stents. The unique bactericidal and anitbiofilm activities of bismuth thiols might contribute to delaying the process of biliary stent occlusion if the effective concentrations of bismuth thiols could be delivered to the target sites. The feasibility of this application of bismuth thiols deserves further investigation.
\end{abstract}

KEY WORDS: stent; biliary; bacteria; bismuth dimercaprol.

Biliary endoprosthesis placement is a common procedure in palliating malignant or benign obstruction of bile tracts, but stent occlusion remains a major problem in this treatment. It is believed that the deposition of calcium salts due to the biochemical activities of bacterial enzymes in the biofilm on the surface of the stents and reflux of intestinal contents into stents are the two main mechanisms of stent occlusion (1-4). Reflux of intestinal contents into

\footnotetext{
Manuscript received July 9, 2004; accepted October 14, 2004

From the *ENH Research Institute, Northwestern University Feinberg School of Medicine, Evanston, Illinois 60201, †Finch University of Health Science, North Chicago, Illinois 60064 , and $¥$ University of Michigan, Ann Arbor, Michigan 48109.

Address for reprint requests: Tat-Kin Tsang MD, Evanston Hospital, Room 1100, 2650 Ridge Avenue, Evanston, Illinois 60201, USA; doctat@aol.com.
}

bile tracts or stents is a prevalent phenomenon when stents are placed across the sphincter of Oddi (32). Besides the direct mechanical blockage caused by large food debris, these small food materials or other tiny amorphous food debris may contribute to stent occlusion by trapping calcium salt deposits in bile, promoting bacterial infection in the stents, or participating in the buildup of sludge. For this mechanism, designing a new stent may be the only solution. For the first mechanism, prevention of bacterial infection and biofilm formation may be of benefit. However, so far no reagents have proven to be satisfactory (5-7).

Due to the antiseptic, astringent, protective, antacid, antisecretory, and local gastrointestinal properties of bismuth (the site of action is within the gastrointestinal lumen), bismuth-containing compounds have been widely used in clinical practice, such as for treatment of dyspepsia and 


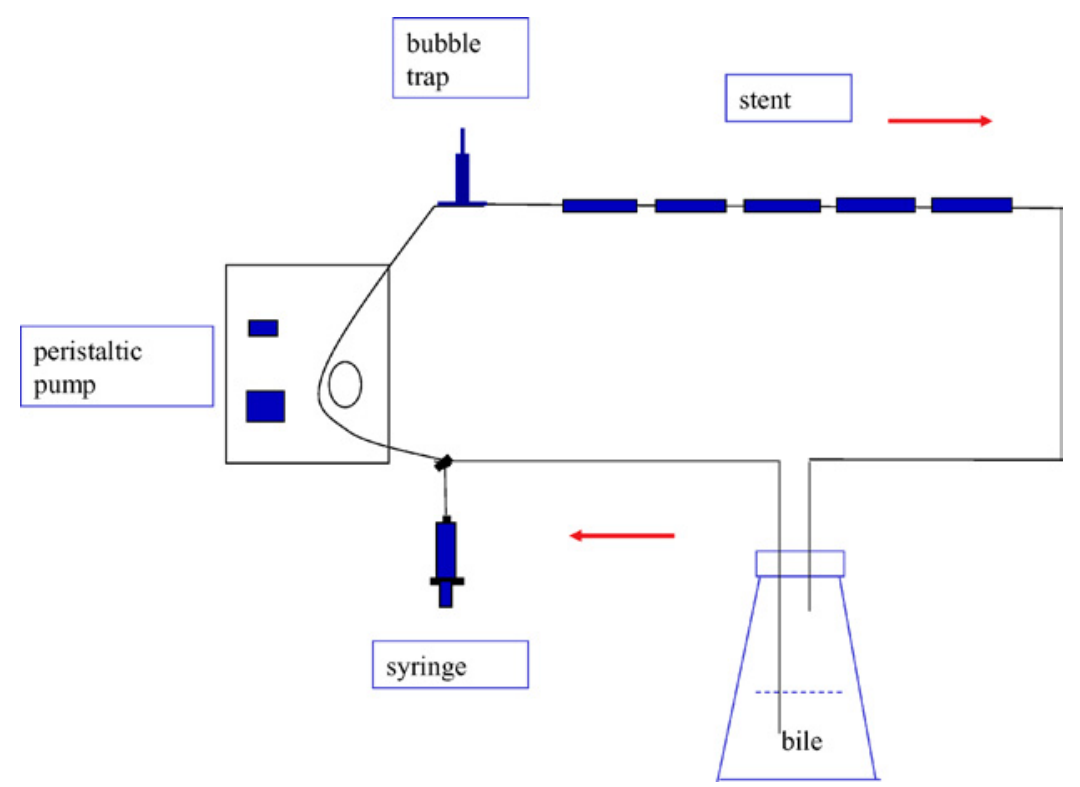

Fig 1. A stent perfusion system in vitro. Five straight, 10-F, 7-cm-long polyethylene stents were connected in tandem in each perfusion circle. Porcine bile was collected and used as the basic perfusion medium. Medium containers were placed in a water bath at $37^{\circ} \mathrm{C}$. Stents were sealed in sterile pouches. Bile flow rate in each perfusion circle was adjusted to $1 \mathrm{ml} / \mathrm{min}$. The pump has multiple channels to drive the perfusion circles.

diarrhea and for the eradication of Helicobacter pylori in the stomach (8-12). Recently, the antibacterial properties of bismuth have been greatly enhanced, up to 1000 -fold, through combination with certain lipophilic thiol compounds, thereby significantly enhancing its potency and versatility as an antibacterial agent (13). A series of reports from Domenico et al. demonstrated the unique effects of bismuth thiols compounds at low concentrations on bacterial virulent factors such as exopolysaccharide expression and bacterial biofilm formation (14-16). Considering the increased antibacterial activities, specific role of antibiofilm at low concentrations, local gastrointestinal property, and relatively low toxicity, we think that bismuth thiols may be a choice for treating bacterial infection and biofilm formation involved in biliary stent occlusion. To explore this possibility and feasibility, this study utilized bismuth-2,3-dimercaptopropanol (BisBAL) as a sample to test the effect of bismuth thiols on the common bacterial strains that attach to biliary stents. This report primarily documents the role of BisBAL in inhibiting the growth of tested bacteria in bile and their effect on the bacterial adherence to the surface of stents in an in vitro bile perfusion system.

\section{MATERIALS AND METHODS}

Bile Perfusion System. A flow cell system (Stovall Life Science, Greensboro, NC) was modified and employed in this study.
Five straight, 10-F, 7-cm-long polyethylene stents (WilsonCook Medical, Winston-Salem, NC) were connected in tandem in each perfusion circle instead of the flow cells, indicated in Figure 1. Porcine bile was collected following the established procedure in our laboratory (17) and used as the basic perfusion medium. Medium containers were placed in a water bath at $37^{\circ} \mathrm{C}$. Stents were sealed in sterile pouches. Bile flow rate in each perfusion circle was adjusted to $1 \mathrm{ml} / \mathrm{min}$ in view of the bile flow rate in the bile duct of human (18).

Previous studies from our laboratory and others indicated that four bacterial species, Escherichia coli, Klebsiella pneumoniae, Enterobacter, and Enterococcus, are the most common microorganisms involved in stent infection $(19,20)$. One strain of each species was used in this study, which were cultured and isolated from the biliary stents that were rermoved from patients. Bacteria were inoculated in bile at about $10^{7}-10^{8}$ colony-forming units $(\mathrm{CFU}) / \mathrm{ml}(21,22)$.

For each bacterial strain, four bile reservoirs $(200 \mathrm{ml}$ each) with additional $0,5,10$, and $20 \mu M$ bismuth BisBAL, were pooled separately in this perfusion system. Bacterial adherence to stent inner surfaces was measured by the platecount method as described below, at 2, 6, 12, 20, and $24 \mathrm{hr}$, respectively.

Preparation of BisBAL. BisBAL was prepared in liquid form according to the method of Domenico et al. (14), by combining bismuth nitrate and dimercaprol (both from Sigma Chemical Co., St. Louis, MO) at a 2:1 molar ratio in propylene glycol. BisBAL was directly diluted into the perfusion medium to the required concentrations.

Demonstration of Bacterial Adherence on Stents. The amount of bacteria adhering to the surfaces of stents was measured using the previously established method (19). In brief, the stents were removed individually at each time point and rinsed 
with $5 \mathrm{ml}$ sterile phosphate-buffered saline (PBS) to remove nonadherent bacteria. The stents were cut into about $0.2-\mathrm{cm}-\mathrm{long}$ portions, and then ultrasonicated in $2 \mathrm{ml}$ PBS to free the adherent bacteria. Serial dilutions of this bacterial suspension were made and cultured on nutrient agar. The results of the overnight cultures were recorded as colony forming units per milliliter of bacterial suspension.

In addition, the amount of bacteria in bile was also measured at each time point by the plate-count method.

Measurement of Slime Products of Bacteria. Most bacteria contain some sort of a polysaccharide layer outside of the cell wall or outer membrane. These extracellular polymeric materials are generally called glycocalyx, but may also be referred to as the bacterial slime layer or exopolysaccharides. To test the ability of the bacterial species included in this study to produce these slime materials, all bacteria were cultured in both nutrient broth and bile for $24 \mathrm{hr}$. Then cultures of bacteria were diluted 1:100 with fresh nutrient broth and 1:50 with bile. Individual wells of sterile, polystyrene, 96-well, flat-bottomed tissue culture plates were filled with $0.2-\mathrm{ml}$ aliquots of the diluted culture. The tissue culture plates were incubated for $24 \mathrm{hr}$ at $37^{\circ} \mathrm{C}$. The contents of each well were gently aspirated by tipping the plate and placing the aspirator tip in the lowest corner of the well. The wells were washed two times with $0.2 \mathrm{ml}$ of PBS. Adherent organisms were fixed in place with Bouin fixative (23) and stained with Hucker crystal violet. Excess stain was rinsed off by placing the plate under running tap water. After drying, the optical density (OD) of stained adherent bacterial films was read at a wavelength of $570 \mathrm{~nm}$ with a Spectra Max 250 plate reader (Global Medical Instrumentation, Inc., Albertville, MN).

Statistical Analysis. The amount of adherent bacteria on the surfaces of stents among different groups was compared using repeated-measures one-way analysis of variance. The differences in slime production of bacteria in different media were analyzed by the nonparametric test. $P$ values less than 0.05 were considered significant for all the tests. All statistical calculations were performed with the use of Prism3.0 (GraphPad Software, Inc., San Diego, CA).

\section{RESULTS}

The ability of each bacterial strain to produce slime was tested in both nutrient broth and porcine bile. The results are shown in Figure 2. When bacteria were cultured in the nutrient broth, the strain of Escherichia coli was the least slime-productive and Enterobacter was the most slime-productive among the four bacterial species, while the strain of Enterococcus produced the least slime but Klebsiella pneumoniae produced the most slime when bacteria were cultured in the bile medium.

The growth curves of bacteria in bile with different concentrations of BisBAL are shown in Figure 3. BisBAL at 5-10 $\mu M$ did not inhibit the growth of Escherichia coli, Enterobacter, and Enterococcus, but $10 \mu \mathrm{M}$ BisBAL inhibited the growth of Klebsiella pneumoniae. BisBAL at $20 \mu M$ was very effective in inhibiting growth for E. coli, Klebsiella pneumoniae, and Enterobacter but not Enterococcus.

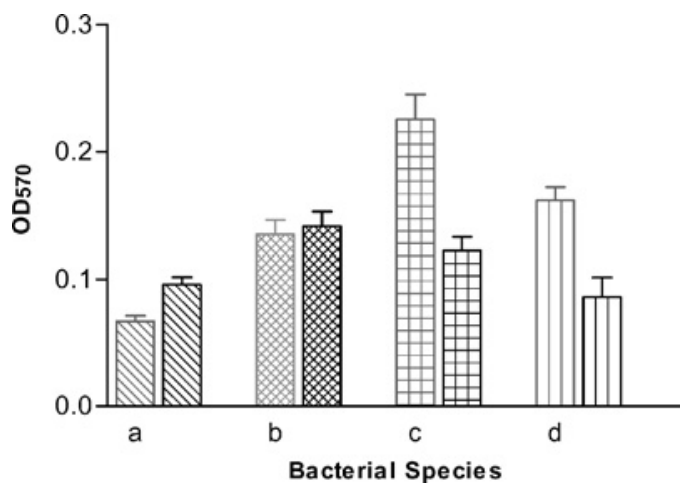

Fig 2. The slime production of each bacterial species growing in nutrient broth (dark bars) and porcine bile (light bars) for $24 \mathrm{hr}$. (a) Escherichia coli; (b) Klebsiella pneumoniae; (c)Enterobacter; (d) Enterococcus. Bacteria adhering to microtiter plates were processed and measured for absorbance at $570 \mathrm{~nm}$. Values are means \pm standard deviations.

The data in Figure 4a show that the amount of Escherichia coli adhered to the surface of stents in the group without BisBAL was significantly higher than that in the group with $20 \mu M$ BisBAL $(P<0.05)$. However, no difference in bacterial adhesion was found among the groups with 0,5 , and $10 \mu M$ BisBAL $(P>0.05)$. When bile was inoculated with Klebsiella pneumoniae as a perfusion medium, as demonstrated in Figure 4b. The addition of $5 \mu M$ BisBAL caused a very remarkable reduction in Klebsiella pneumoniae adherence to the surface of the stents. At $10 \mu M$, BisBAL in bile did not cause a greater reduction of bacterial adherence to the surface of stents than at $5 \mu M$. However, $20 \mu M$ BisBAL in bile decreased bacterial adherence to the surface of the stents even further. Similarly, less bacterial adhesion of Enterobacter was seen with higher concentrations of BisBAL, as indicated in Figure 4c. When Enterococcus was tested, as shown in Figure 4d, the amount of bacteria adhering to the stent surface was less in the group with $5 \mu M$ BisBAL than the group without BisBAL. However, adding more BisBAL to the bile in the other two groups did not further reduce the number of bacteria adhering to the surface of stents.

\section{DISCUSSION}

Under normal conditions, duodenal refluxing to the common bile duct and pancreatic duct do not usually occur, so bile and pancreatic juice are sterile $(24,25)$. In the treatment of malignant or benign obstructive jaundice for inoperable cases, it is conventional to place a plastic biliary stent across the sphincter of Oddi. As a result of the stenting across the sphincter of Oddi, low pressure in the common bile duct and breakage of the bacterial barrier facilitate the reflux of intestinal contents and ascending 


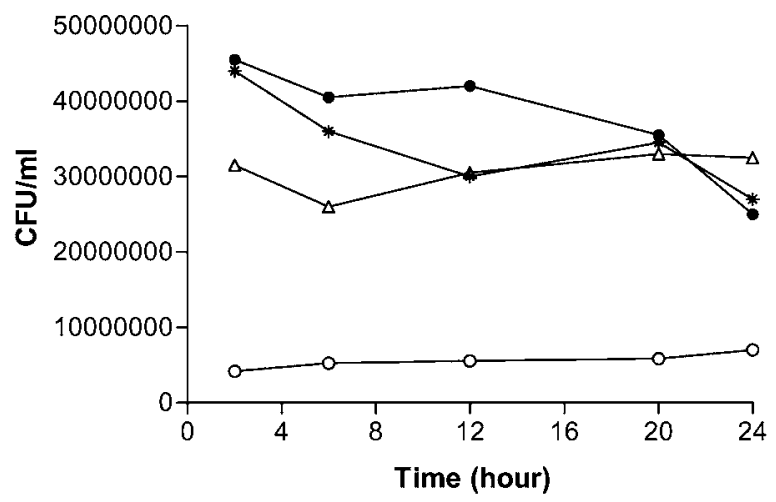

(a)

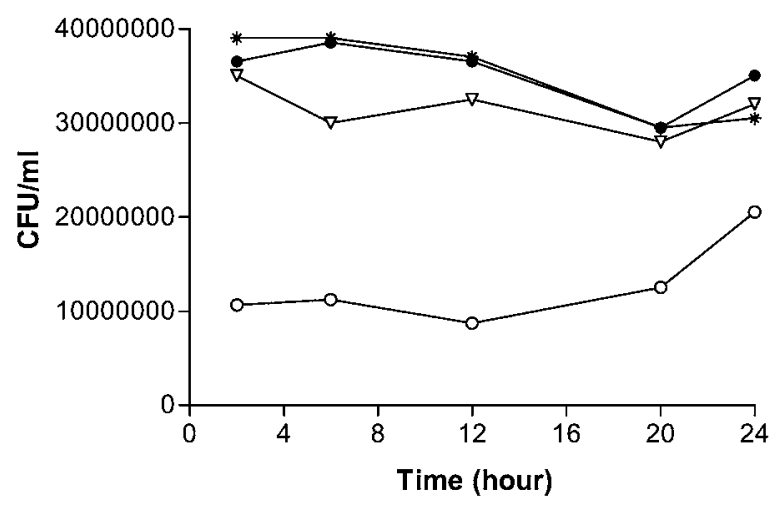

(c)

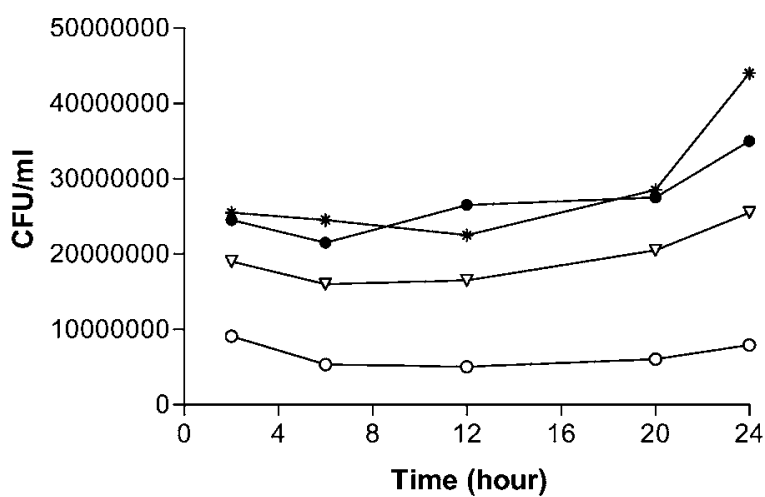

(b)

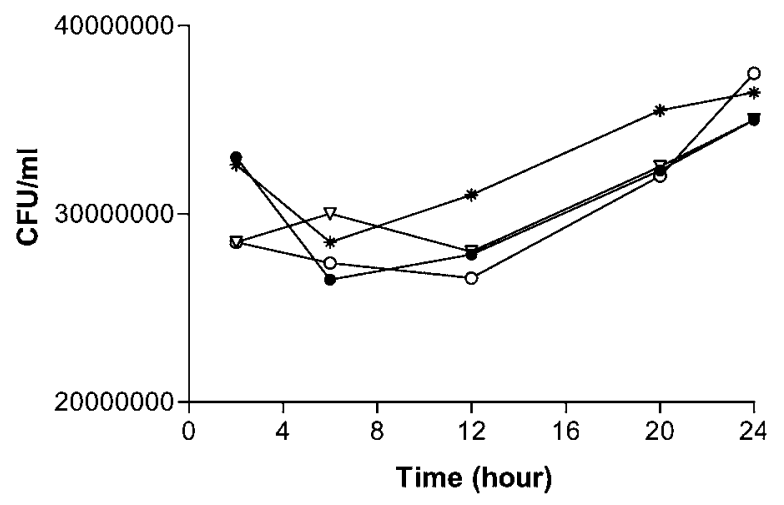

(d)

Fig 3. Growth curves of bacteria in bile with BisBAL at different concentrations: $0 \mu M(*), 5 \mu M(\bullet), 10 \mu M(\Delta)$, and $20 \mu M(0)$. (a) Escherichia coli; (b) Klebsiella pneumoniae; (c) Enterobacter; (d) Enterococcus. Bacteria were measured with the plate-count method and are expressed as colony forming units per milliliter of bacterial suspension. Values are means of double determinations.

bacterial infection $(26,27)$. While the stenting procedure is performed, bacteria in the intestinal tract may also be brought into the bile duct. Moreover, when a plastic stent is placed in the bile duct, the bacteria in bile easily attach, colonize, and grow on the inner surface of the stent. Since most bacterial species can produce slime, a bacterial biofilm is often formed on the stent surface. Persistent existence of bacterial biofilm and consequent deposition of salts for bacterial enzymatic activities result in the final failure of most biliary stenting treatments.

Bacterial biofilm is a special microorganism community, in which bacteria respond differently to antimicrobials compared to suspended bacteria. Biofilm bacteria may express some genes that generate antimicrobial resistance. Biofilms may exhibit heterogeneity such as gradients in physiology where slower-growing organisms may be more resistant to antimicrobials. Bacteria are usually surrounded by the extracellular matrix and biofilms in the field may accumulate inorganic or organic substances from the surrounding medium; as a result, antimicrobials may not fully penetrate a biofilm (28-30). Therefore, to prevent the complications and improve the effectiveness of biliary stents, it is critical to control bacterial infection before bacterial biofilm has formed on the surface of stents. Some strategies, such as preventing bacteria from entering the bile duct, killing the suspended bacteria in bile, or inhibiting initial bacterial adherence, could be taken. This study showed the particular effect of BisBAL at different concentrations on the tested bacteria in vitro. Those specific functions of BisBAL may be employed in preventing bacterial biofilm formation on the surface of stent.

The bactericidal effect of bismuth thiols in the bile or in the biliary tract system has never been investigated. This study addressed the information. The results showed that BisBAL at $10 \mu M$ significantly decreased the amount of Klebsiella pneumoniae growing in bile, the effects of BisBAL at $20 \mu M$ are more intensive, and it largely reduced the amount of another two bacterial strains: Escherichia coli and Enterobacter. The activities of bismuth thiols favor a high temperature and $\mathrm{pH}$ value. In our study the $\mathrm{pH}$ value of porcine bile was approximately $7.0(6.90 \pm 0.48)$, and the system temperature was set at $37^{\circ} \mathrm{C}$ to simulate 


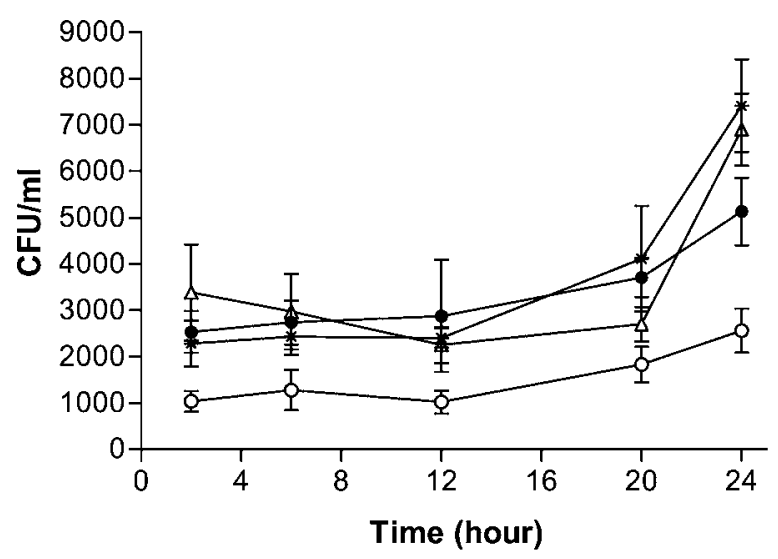

(a)

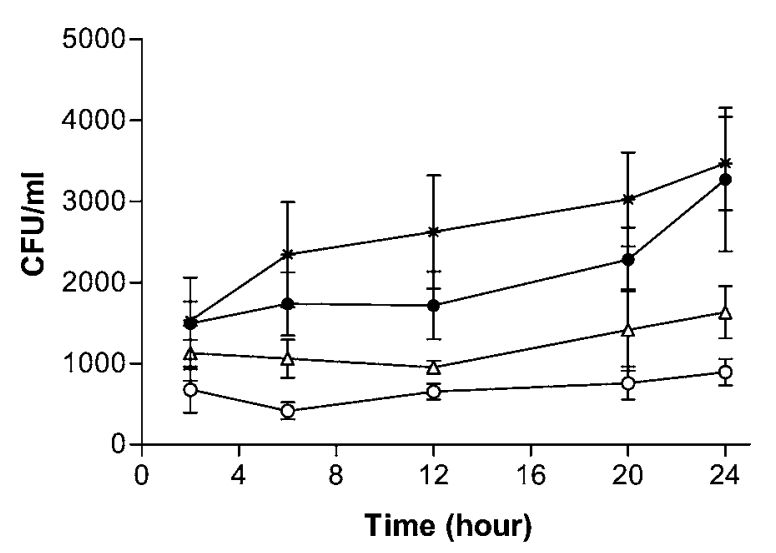

(c)

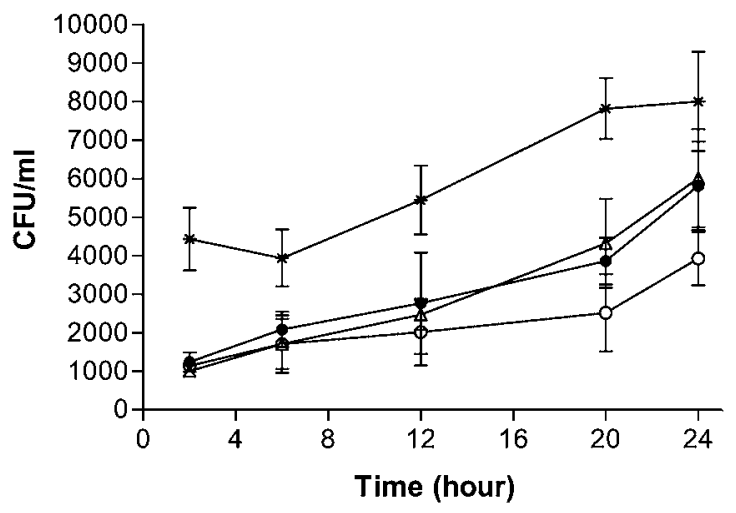

(b)

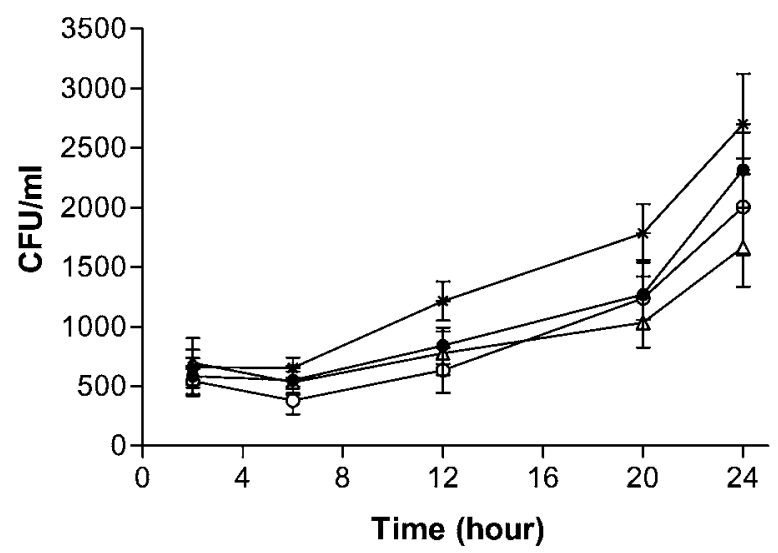

(d)

Fig 4. Bacteria adhering to the surface of stents when stents were perfused with bile with different concentrations of BisBAL: $0 \mu M(*), 5 \mu M(\bullet), 10 \mu M(\Delta)$, and $20 \mu M(0)$. (a) Escherichia coli; (b) Klebsiella pneumoniae; (c) Enterobacter; (d) Enterococcus. Bacteria were measured with the plate-count method and are expressed as colony forming units per milliliter of bacterial suspension.

the human body temperature. The above results indicated that BisBAL was functioning in porcine bile at the experimental temperature in vitro. Since the $\mathrm{pH}$ value and compositions of porcine bile are similar to those of human bile, we believe that BisBAL could function as a bactericidal reagent against certain bacteria in the human biliary system in vivo if it could be supplied at the effective concentrations.

Yet a more interesting finding from our study was that BisBAL at low concentration $(5 \mu M)$ reduced Klebsiella pneumoniae, Enterobacter, and Enterococcus adherence to the surface of biliary stents in vitro. Previous studies have demonstrated that subinhibitory levels $(3-5 \mu M)$ of BisBAL can inhibit expression of glycocalyx or exopolysaccharide in Klebsilla pneumoniae, Staphylococca, and Pseudmonas aeruginosa in certain culture broths (1416, 31). These bacterial surface structures, such as capsules, flagella, and fimbriae (or pili), as well as slime, along with the physical properties of substratum surfaces, are the most important factors for bacterial attachment and adherence to a surface (28). It is unsure whether the effect of BisBAL at a low concentration $(5 \mu M)$ against bacterial adherence to the surface of biliary stents in this study is through inhibition of the expression of glycocalyx or exopolysaccharide as in other culture media in previous studies. This is because bacteria might present different biological behaviors in different culture media. As demonstrated by our results, the four bacterial strains changed their ability to produce slime when the culture medium was switched from nutrient broth to porcine bile. Moreover, BisBAL may have different effects on the same bacteria in different environments. The interactions among BisBAL, bacteria, and bile compositions are complicated. Despite the uncertain mechanism, this study demonstrated with certainty that BisBAL at a low concentration $(5 \mu M)$ could inhibit the adherence of certain bacteria to the inner surface of biliary stents, which might contribute to control biofilm formation in the initial process. 
As demonstrated in this study, $5 \mu M$ BisBAL did not affect the adhesion of the tested $E$. coli strain to the surface of stents and $20 \mu M$ BisBAL did not inhibit the growth of tested Enterococcus. As reported in one study (20), a lot of bacterial species and strains have been isolated from biliary stents removed from patients. To ensure that bismuth thiols are effective and broad-spectrum enough as antibacterial reagents against the bacteria involved in biofilm formation on biliary stents, further work needs to be done, including tests with more bacterial species and strains and with wider ranges of concentration of bismuth thiols. Routes of application of bismuth thiols for controlling bacterial infection in biliary stents, such as coating the stent surface with special materials with bismuth thiols incorporated, need to be tested in vivo. In short, the mechanism of bismuth thiols to prevent bacterial biofilm formation on the surface of biliary stents and the feasibility of applying it to reduce the complications of biliary stenting need to be investigated further.

\section{REFERENCES}

1. Speer AG, Cotton PB, Rode J, et al.: Biliary stent blockage with bacterial biofilm: a light and electron microscopy study. Ann Intern Med 108(4):546-553, 1988

2. Wosiewitz U, Schrameyer B, Safrany L: Biliary sludge: its role during bile drainage with an endoprosthesis. Gastroenterology 88:1706, 1985

3. Sung JY, Leung JW, Shaffer EA, Lam K, Costerton JW: Bacterial biofilm, brown pigment stone and blockage of biliary stents. J Gastroenterol Hepatol 8(1):28-34, 1993

4. Moesch C, Sautereau D, Cessot F, et al.: Physicochemical and bacteriological analysis of the contents of occluded biliary endoprostheses. Hepatology 14(6):1142-1146, 1991

5. Leung JW, Libby ED, Morck DW, et al.: Is prophylactic ciprofloxacin effective in delaying biliary stent blockage? Gastrointest Endosc 52(2):175-182, 2000

6. Rees EN, Tebbs SE, Elliott TS: Role of antimicrobial-impregnated polymer and Teflon in the prevention of biliary stent blockage. J Hosp Infect 39(4):323-329, 1998

7. Leung JW, Lau GT, Sung JJ: Decreased bacterial adherence to silver-coated stent material: An in vitro study. Gastrointest Endosc 38(3):338-340, 1992

8. Tillman LA, Drake FM, Dixon JS. Wood JR: Review article: safety of bismuth in the treatment of gastrointestinal diseases. Aliment Pharmacol Ther 10(4):459-467, 1996

9. Kappstein I, Engels I: Antibacterial activity of sucralfate and bismuth subsalicylate in simulated gastric fluid: Eur J Clin Microbiol 6(2):216-217, 1987

10. De Boer WA: Bismuth triple therapy: still a very important drug regimen for curing Helicobacter pylori infection. Eur J Gastroenterol Hepatol 11(7):697-700, 1999

11. Coelho LG, Passos MC, Chausson Y, Castro L de P: Five-day bismuth-free triple therapy for the eradication of Helicobacter pylori and reduction of duodenal ulcer relapse. Am J Gastroenterol 86(8):971-975, 1991
12. Farthing MJ: Review article: prevention and treatment of travellers' diarrhoea. Aliment Pharmacol Ther 5(1):15-30, 1991

13. Domenico P, Salo RJ, Novick SG, Schoch PE, Van Horn K, Cunha BA: Enhancement of bismuth antibacterial activity with lipophilic thiol chelators. Antimicrob Agents Chemother 41(8):1697-1703, 1997

14. Domenico P, Tomas JM, Merino S, Rubires X, Cunha BA: Surface antigen exposure by bismuth dimercaprol suppression of Klebsiella pneumoniae capsular polysaccharide. Infect Immun 67(2):664-669, 1999

15. Domenico P, Baldassarri L, Schoch PE, Kaehler K, Sasatsu M, Cunha BA: Activities of bismuth thiols against staphylococci and staphylococcal biofilms. Antimicrob Agents Chemother 45(5):1417-1421, 2001

16. Huang CT, Stewart PS: Reduction of polysaccharide production in Pseudomonas aeruginosa biofilms by bismuth dimercaprol (BisBAL) treatment. J Antimicrob Chemother 44(5):601-605, 1999

17. Chodash HB, Tsang TK, Pollack JM, Eisenman RE, Rege RM, Ostrow JD: Effect of temperature on stability of eight components of porcine gallbladder bile. Dig Dis Sci 42(2):273-278, 1997

18. Howard PJ, Murphy GM, Dowling RH: Gallbladder emptying patterns in response to a normal meal in healthy subjects and patients with gallstones: ultrasound study. Gut 32:1406-1411, 1991

19. Zhang HJ, Tsang TK, Jack CA: Bile glycoprotein mucin in sludge occluding biliary sent. J Lab Clin Med 142(1):58-61, 2003

20. Molinari G, Pugliese V, Schito GC, Guzman CA: Bacteria involved in the blockage of biliary stents and their susceptibility to antibacterial agents. Eur J Clin Microbiol Infect Dis 15(1):88-92, 1996

21. Zhang HJ, Tsang TK, Jack CA, Pollack JM: Role of bile mucin in bacterial adherence to biliary stent. J Lab Clin Med 139(1):28-34, 2001

22. Flores C, Maguilnik I, Hadlich E, Goldani LZ: Microbiology of choledochal bile in patients with choledocholithiasis admitted to a tertiary hospital. J Gastroenterol Hepatol 18(3):333-336, 2003

23. Baldassarri L, Simpson WA, Donelli G, Christensen GD: Variable fixation of staphylococcal slime by different histochemical fixatives. Eur J Clin Microbiol Infect Dis 12(11):866-868, 1993

24. Torsoli A: Physiology of the human sphincter of Oddi. Endoscopy 20:166-170, 1998

25. Sung JY, Costerton JW, Shaffer EA: Defense system in the biliary tract against bacterial infection. Dig Dis Sci 37(5):689-696, 1992

26. Sung JY, Leung JW, Shaffer EA, Lam K, Costerton JW: Ascending infection of the biliary tract after surgical sphincterotomy and biliary stenting. J Gastroenterol Hepatol 7(3):240-245, 1992

27. Pederson FM, Lassen AT, de Muckadell OBS: Randomized trial of stent placed above and across the sphincter of Oddi in malignant bile duct obstruction. Gastrointest Endosc 48:574-579, 1998

28. An YH, Friedman RJ: Concise review of mechanisms of bacterial adhesion to biomaterial surfaces. J Biomed Mater Res 43(3):338348,1998

29. Costerton JW, Cheng KJ, Geesey GG, et al.: Bacterial biofilms in nature and disease. Annu Rev Microbiol. 41:435-464, 1987

30. Costerton JW, Stewart PS, Greenberg EP: Bacterial biofilm: A commom cause of persistent infections. Science 284:1318-1322, 1999

31. Wu CL, Domenico P, Hassett DJ, Beveridge TJ, Hauser AR, Kazzaz JA: Subinhibitory bismuth-thiols reduce virulence of Pseudomonas aeruginosa. Am J Resp Cell Mol Biol 26(6):731-738, 2002 